\title{
A low-threshold, high-efficiency microfluidic waveguide laser
}

Dmitri V. Vezenov, Brian T. Mayers, Richard Conroy and George M. Whitesides

Department of Chemistry and Chemical Biology, Harvard University, 12 Oxford St., Cambridge, MA 02138

and

Preston Snee, Yinthai Chan, Daniel G. Nocera, and Moungi G. Bawendi

Department of Chemistry, Massachusetts Institute of Technology,

77 Massachusetts Ave., Cambridge MA 02139

\section{Supplemental information}

\section{Details of fabrication of microfluidic waveguide lasers and their emission characteristics}

March 29, 2005 


\section{Materials and Methods}

Fabrication of microfluidic devices. The microfluidic channels were fabricated as in several previous publications. ${ }^{1-3}$ High-resolution chrome masks were designed in Clewin (WieWeb Software, www.wieweb.com) and printed with laser-beam writing. The photoresist masters were made of SU-8-100 (Microchem, 3000 RPM; 100- $\mu$ m thick) on silicon wafers (Silicon Sense, Inc, Nashua, NH) by standard photolithography with chrome masks. The surface of the photoresist master and silicon wafer were coated with tridecafluoro-1,1,2,2- tetrahydrooctyl-1- trichlorosilane (United Chemical Technology, PA) to lower the surface free energy; this coating allowed the poly(dimethylsiloxane) (PDMS, Dow, Sylgard 184) replica to be removed without damaging the master. PDMS was poured directly onto the master and cured for 2 hours at $60^{\circ} \mathrm{C}$. The PDMS replica was removed under methanol to prevent damage to the features on the replica. A homemade punch was used to drill holes in the inlets and the outlets before sealing of the channels.

Mirrors were evaporated onto the PDMS replica at the ends of the waveguide using electron beam evaporation (VEECO). The PDMS replica was placed on the evaporation stage at a $45^{\circ}$ angle (see Figure S1). This orientation allowed one end of the device to be metal-coated; rotating the PDMS replica by $180^{\circ}$ allowed the other end of the device to be metal-coated. Both ends of the waveguide were thus coated with an adhesion layer of $\mathrm{Ti}(5 \mathrm{~nm})$ and a layer of Au. The thickness of the gold layer determined the final reflectivity of the mirror. In a typical device, one end was coated 
Figure S1. Mirrors were fabricated at the opposing ends of the waveguide by electronbeam deposition of a layer of $\mathrm{Ti}(5 \mathrm{~nm})$ and $\mathrm{Au}(40-100 \mathrm{~nm})$. The mirrors were deposited on ends of the channels by evaporating onto the device twice at a $45^{\circ}$ angles. (A) Depicts evaporation of the $40 \mathrm{~nm}$ gold layer onto the downstream T-junction of the device. (B) Depicts evaporation of the $100 \mathrm{~nm}$ gold layer onto the upstream T-junction. The excess gold is removed with "Scotch tape", leaving only the gold that was evaporated on the recessed portions of the waveguide.

\section{Figure S1}

A

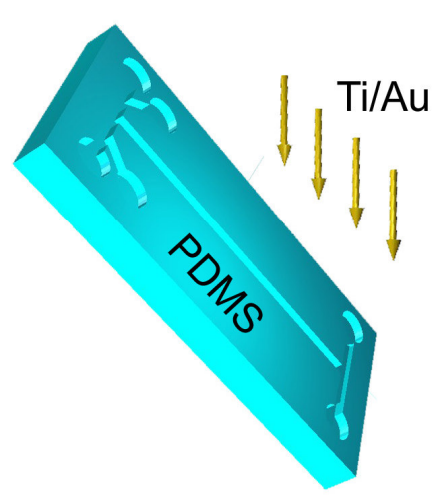

B

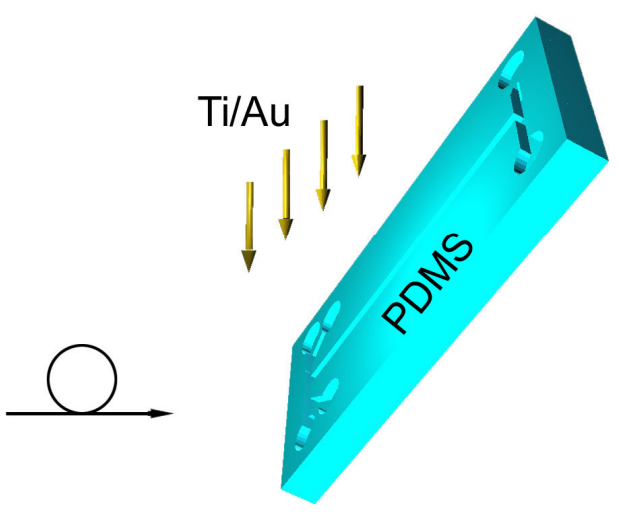


with $40 \mathrm{~nm}$ of $\mathrm{Au}$ ( $70 \%$ reflectivity), and the other with $100 \mathrm{~nm}$ of $\mathrm{Au}(\sim 85 \%$ reflectivity). The excess gold on the surface of the PDMS replica was easily removed with a piece of "Scotch" tape that was brought into contact with the surface. The upraised gold preferentially adhered to the sticky side of the scotch tape, while the gold in the recessed areas remained on the PDMS.

Microfluidic channels were prepared by sealing the PDMS replica of the photoresist master to a flat piece of PDMS. The PDMS replica and the flat piece of PDMS were exposed to oxygen plasma (Harrick PDC, Harrick, PA) for $60 \mathrm{~s}$. The exposed surfaces were brought into contact with each other manually and allowed to seal. The sealed channels were placed in a quartered Petri dish with the output face placed $\sim 2$ $\mathrm{mm}$ from the plastic dividers in the dish. Another layer of PDMS was poured around the sealed channels and cured for 2 hours at $60^{\circ} \mathrm{C}$. This layer of PDMS conformed to the plastic divider in the petri dish producing a flat surface for the laser output.

$\mathbf{L}^{2}$ waveguides. We chose rhodamine 640 perchlorate as our standard dye to characterize the device because of it's widespread use in dye lasers. We used methanol $\left(\mathrm{n}_{\mathrm{D}}=1.323\right)$, ethylene glycol $\left(\mathrm{EG}, \mathrm{n}_{\mathrm{D}}=1.431\right)$, and methylsulfoxide $\left(\mathrm{DMSO}, \mathrm{n}_{\mathrm{D}}=1.479\right)$ as solvents to provide a range of refractive indices with which we formed our $\mathrm{L}^{2}$ waveguides. Liquids were driven through the microfluidic device by syringe pumps (Genie, Kent Scientific Corporation, CT) at rates from 32 to $4 \mathrm{~mL} / \mathrm{h}$ (Reynolds number, $\mathrm{Re}, \sim 0.001-0.02)$. The waveguide comprised the long axis of the device, bound by two T-junctions, in which the two cladding flows (of methanol) sheathed the dye-containing central core flows (of methanol, EG, or DMSO). Smooth, linear interfaces between the 
core and cladding fluids were necessary for low-loss guiding of light. Roughness (r) in the walls of the PDMS channel does not affect the interface of the two liquids as long as the width of the flowing cladding streams are each $>2$.

Optical excitation and characterization. The $\mathrm{L}^{2}$ waveguides were optically pumped with a 532-nm laser beam (frequency doubled Nd:YAG, $50 \mathrm{~Hz}$ repetition rate, 16 ns pulse) which was elongated with a cylindrical lens. The optical pumping region covered a 10-mm region of the the microfluidic waveguide (the conversion efficiency was halved in 5-mm long channel, and dropped by an order of magnitude in a $20 \mathrm{~mm}$ long channel as expected for a $10 \mathrm{~mm}$ long excitation beam, see Figure S3). The scheme (Figure 1, main text) displays the configuration of the device with gold mirrors on the opposing T-junctions. Beyond the downstream T-junction, the device terminated with a flat window. A lens collected the laser radiation and focused it onto an optical fiber for input into spectrometer $(0.2 \mathrm{~nm}$ resolution, USB2000, Ocean Optics, WwW.oceanoptics.com). 
Table S1. Transit time of the dye in $10 \mathrm{~mm}$ long microfluidic channel (400 $\mu \mathrm{m} \times 100 \mu \mathrm{m}$ cross-section).

\begin{tabular}{ccc}
$\begin{array}{c}\text { total flow rate } \\
\mathrm{mL} / \mathrm{h}\end{array}$ & $\begin{array}{c}\text { linear velocity } \\
\mathrm{m} / \mathrm{s}\end{array}$ & $\begin{array}{c}\text { channel transit time } \\
\mathrm{s}\end{array}$ \\
\hline 4 & 0.0278 & 0.36 \\
8 & 0.0556 & 0.18 \\
12 & 0.0833 & 0.12 \\
16 & 0.111 & 0.0901 \\
32 & 0.222 & 0.0450 \\
64 & 0.444 & 0.0223
\end{tabular}


Figure S2. Effect of diffusion on distribution of refractive index within microfluidic channel (10 mm long and $400 \mu \mathrm{m}$ x $100 \mu \mathrm{m}$ cross-section, core: DMSO, cladding: methanol): top view of two-dimensional refractive index distribution in the channel for the total flow rate of $4 \mathrm{~mL} / \mathrm{h}(\mathrm{A})$ and $16 \mathrm{~mL} / \mathrm{h}(\mathrm{B})$ for each inlet (we used diffusion coefficient of $10^{-9} \mathrm{~m}^{2} / \mathrm{s}$ in these calculations).
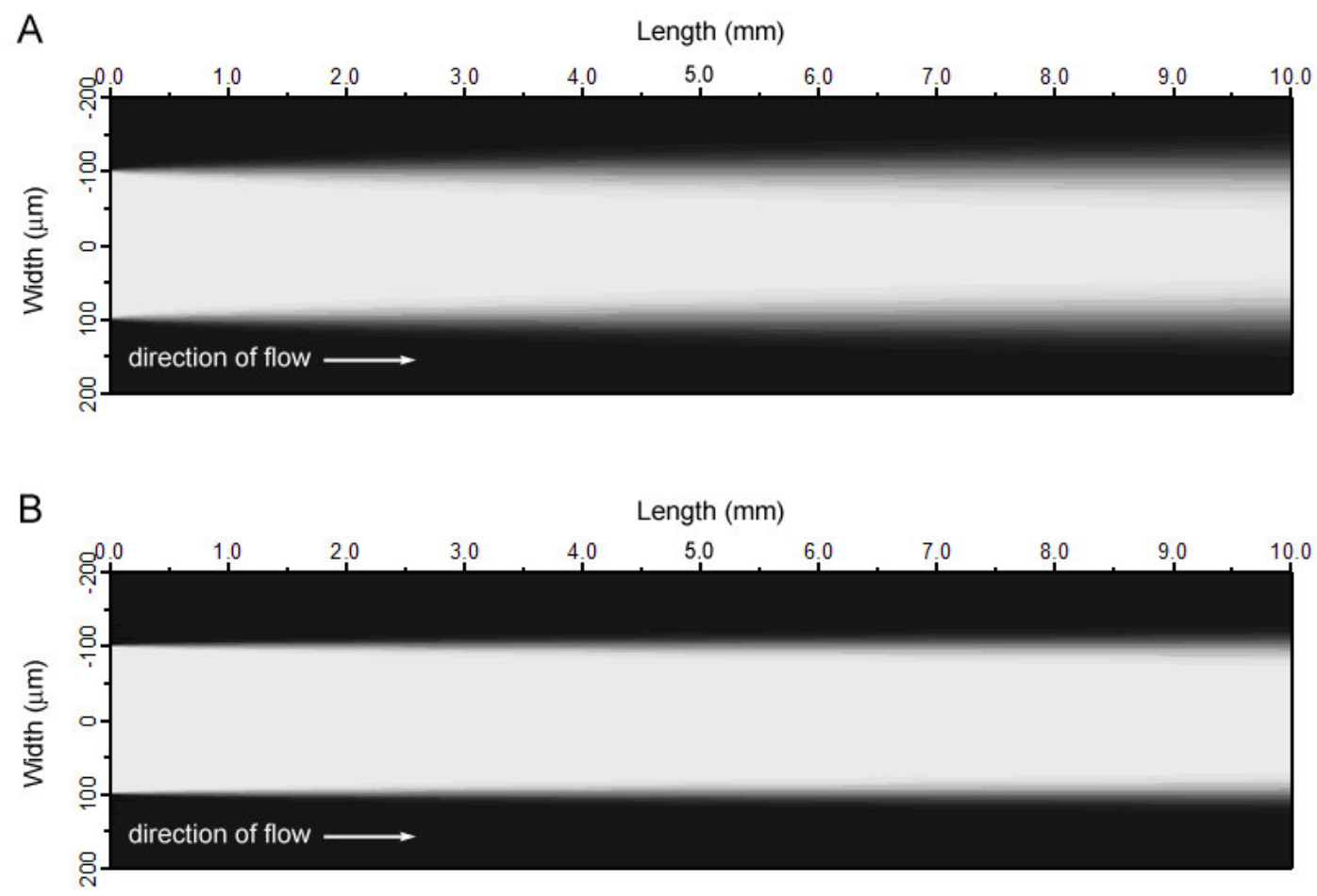

\section{Refractive index:}

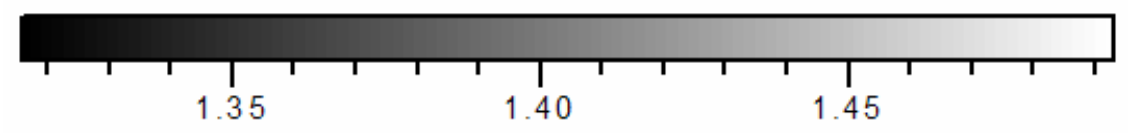


Figure S3. Intensity of the output light versus input power for microchannels of different lengths. In each case, the system employed a solution of $2 \mathrm{mM}$ rhodamine 640 perchlorate in ethylene glycol as the core and methanol as the cladding.

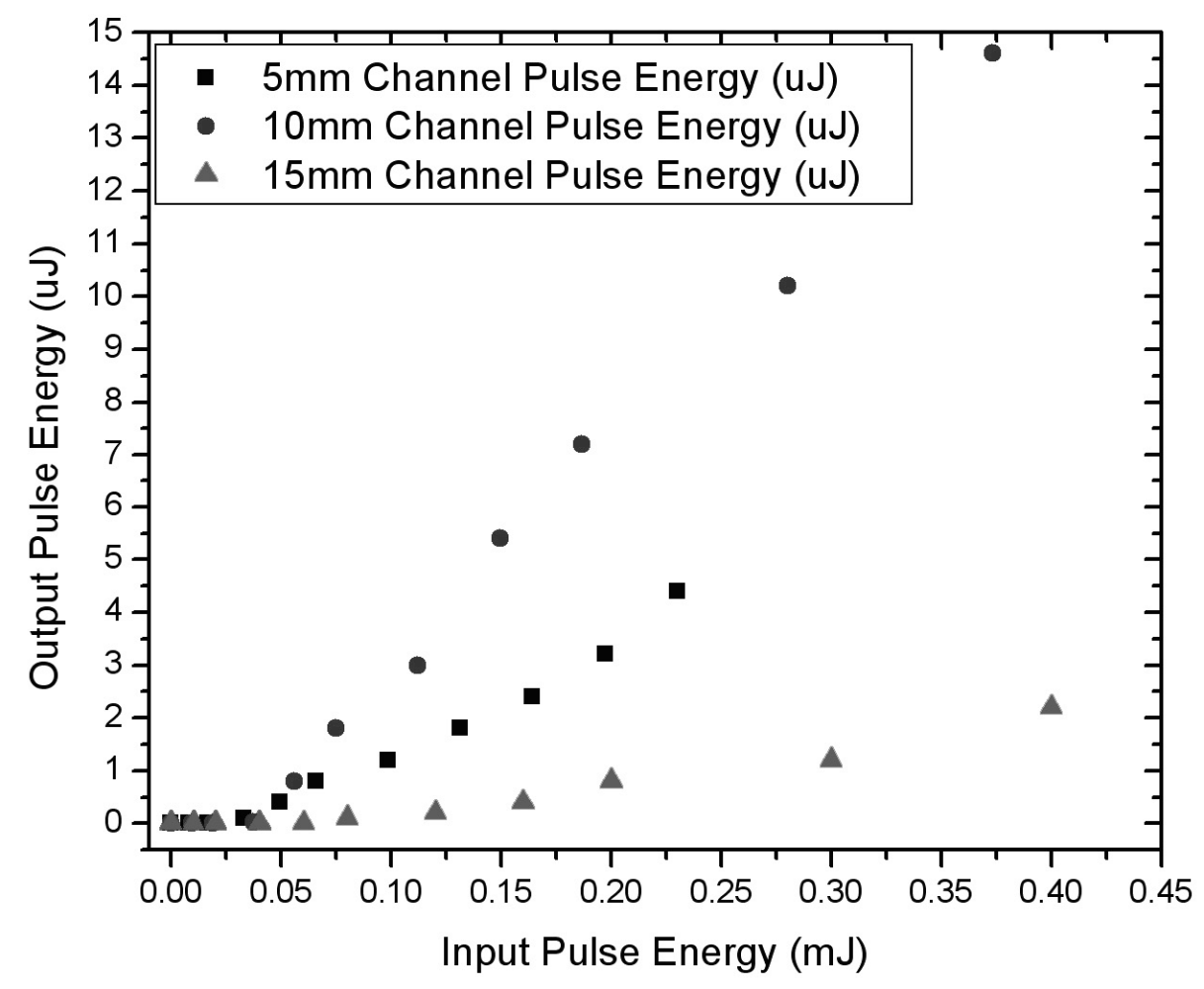


Figure S4. ASE in $\mathrm{L}^{2}$ waveguides (devices without terminal mirrors). (A) Emission spectra at different pump powers measured on-axis (note the $10^{4}$ difference in scale for spectra at input powers below (dashed) and above (solid) threshold). (B) Output peak linewidth versus input pulse energy for $10 \mathrm{~mm} \mathrm{~L}{ }^{2}$ waveguides having methanol and ethylene glycol liquid core and methanol liquid cladding. The curves are sigmoidal fits to guide the eye. (C) Output intensity (measured as peak height) for device having methanol core and cladding. In all cases, the core contained 2-mM rhodamine 640 perchlorate.
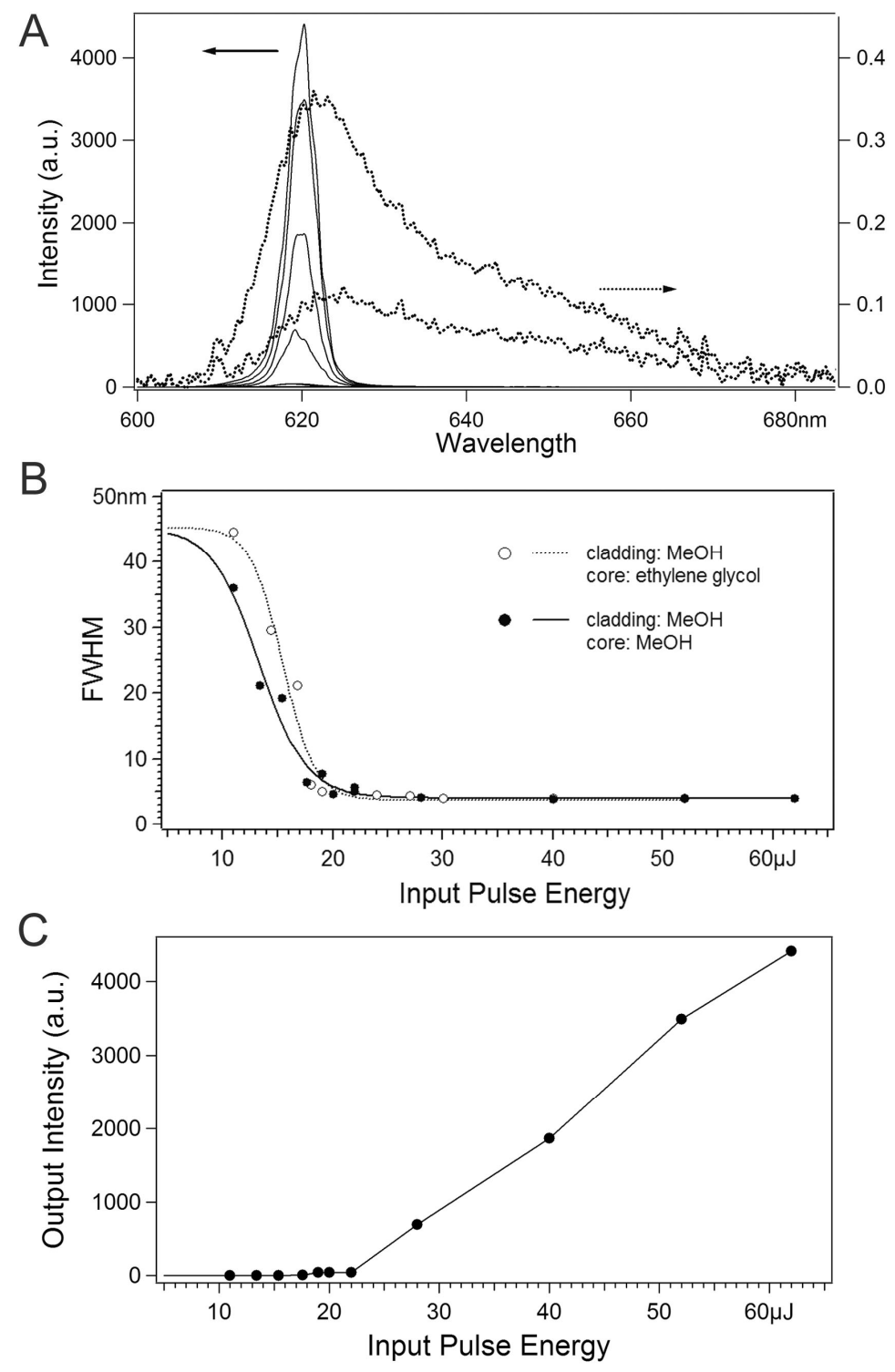
Figure S5. Peak wavelength (black) and lasing threshold (red) for different concentrations of rhodamine 640 perchlorate in a $\mathrm{L}^{2}$ waveguide with gold mirrors, with ethylene glycol as the core and methanol as the cladding.

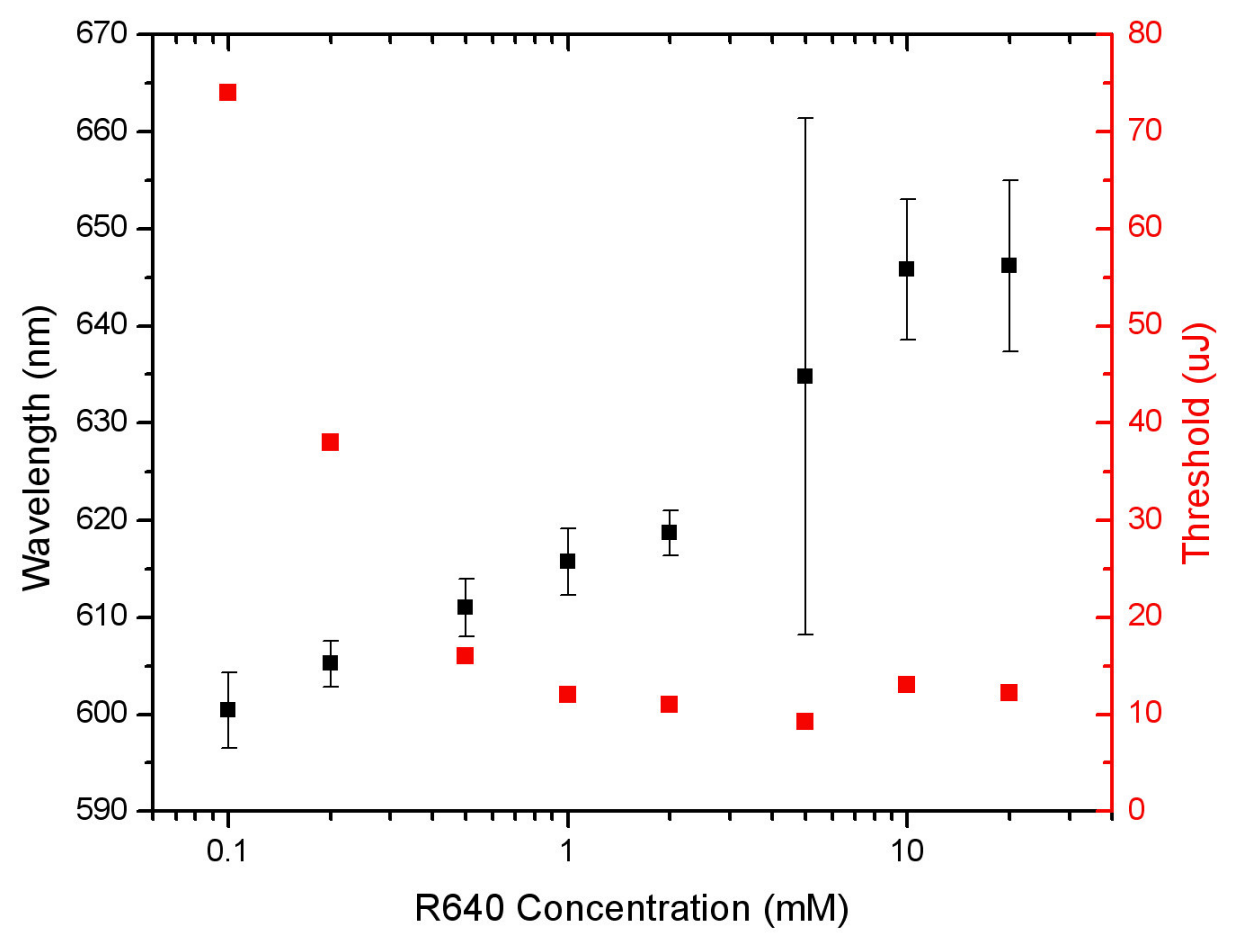




\section{Description of ASE in $\mathbf{L}^{2}$ waveguides.}

For our $\mathrm{L}^{2}$ waveguide laser, the behavior of the ASE in the low and high gain saturation regimes can be described using analytical relationships given by Svelto at el., who considered an active medium operating in a four-level scheme in a geometry given in the Figure S5. Since most ASE emission arises from elements near $\mathrm{z}=0$, i.e. for $\Omega(\mathrm{z})=\Omega(0)=\Omega$, for intensities, $I$, below the characteristic saturation intensity of the laser dye transition, $I_{s}=h v_{0} / \sigma_{\mathrm{p}} \tau$ (where $h$ is Plank's constant, $v_{0}$ is the peak transition frequency, $\sigma_{\mathrm{p}}$ is the peak cross-section, and $\tau$ is the lifetime of the transition), the total ASE intensity is given by:

$$
I=\phi I_{s}\left(\frac{\Omega}{k}\right) \frac{(G-1)^{3 / 2}}{(G \ln G)^{1 / 2}},
$$

where $\mathrm{k}=4(\pi)^{3 / 2}$ for Lorentzian line, or $\mathrm{k}=4 \pi$ for Gaussian line, $\phi$ is the quantum yield and $\mathrm{G}=\exp \left(\sigma_{\mathrm{p}} \mathrm{NL}\right)$ is the peak unsaturated gain of the amplifying medium ( $\mathrm{N}$ is the inversion, $\mathrm{L}$ is the length of the optical path in the channel). IN a deep saturation regime, a simple asymptotic expression is obtained by assuming that half of available fluorescence power is contained in the beam propagating in one direction (and the other half - in the beam in the opposite direction):

$$
\mathrm{I}=\frac{1}{2} \phi \mathrm{I}_{\mathrm{s}} \ln \mathrm{G}
$$

We fitted the data in Figure $S 6$ to equations $\mathrm{S} 1$ and $\mathrm{S} 2$ by assuming $\mathrm{N} \propto \mathrm{I}_{\text {pump. }}$. The fitting described the experimental values well and led to a value of $\Omega \sim 0.007$ steradian (or $\sim 80$ miliradians) for beam divergence. 
Figure S5. (A) Geometry of the active medium for the ASE calculation. $\Omega(\mathrm{z})$ is the solid angle subtended by the exit facet of the medium (at $\mathrm{z}=\mathrm{L}$ ) by active medium element dz. (B) Effect of waveguiding on divergence angle of ASE emission $\left(n_{1}\right.$ and $n_{2}$ is the index of refraction of the liquid cladding and liquid core).

A

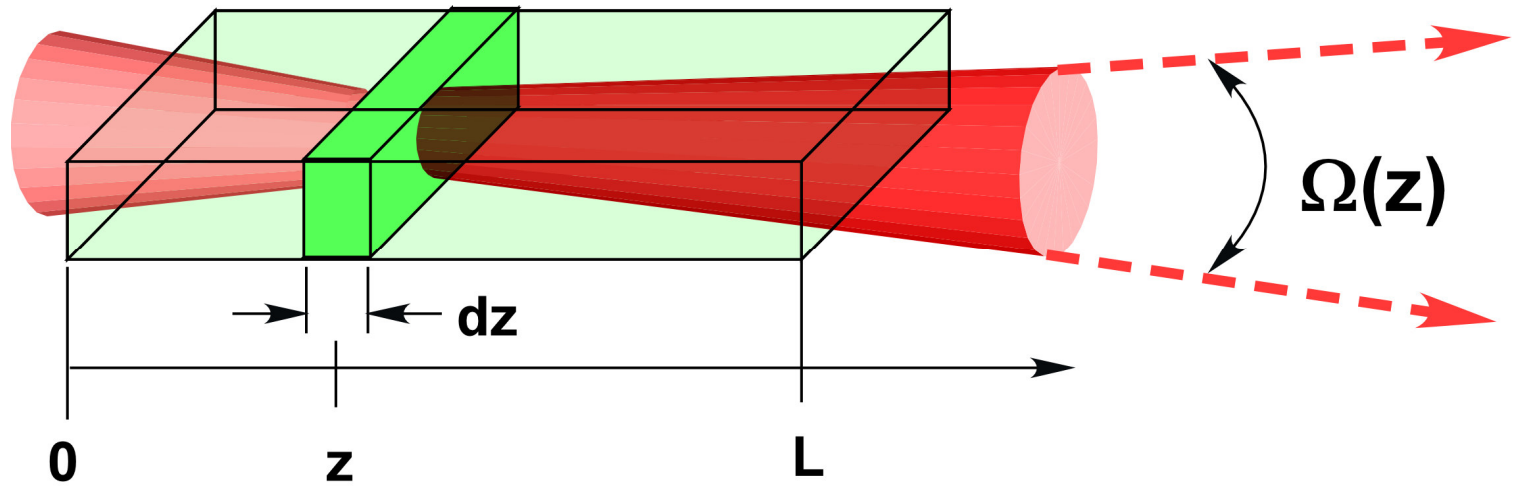

B

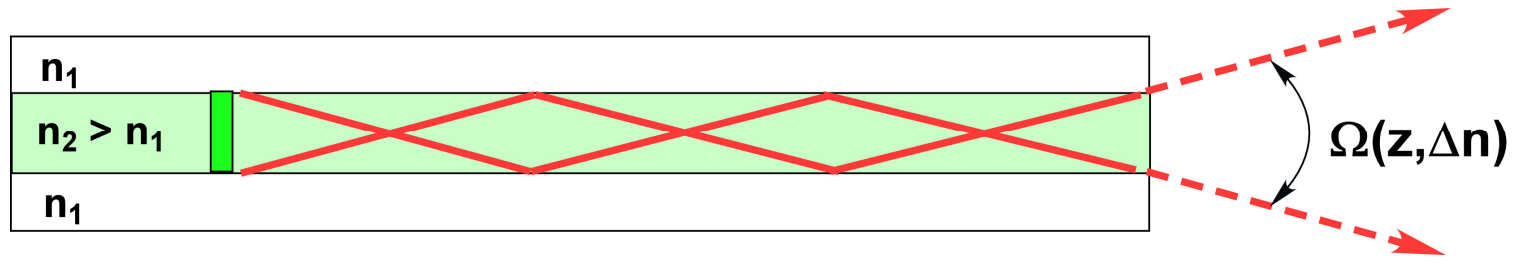


Figure S6. ASE in $\mathrm{L}^{2}$ waveguides containing 2-mM rhodamine 640 perchlorate (devices without terminal mirrors). Output pulse energy (A) and peak width (B) versus input pulse energy for $10 \mathrm{~mm} \mathrm{~L}{ }^{2}$ waveguides having liquid core of different refractive index. In (A), the curves are fits to two limiting regimes (below and above saturation for transition in laser dye) for methanol used as both liquid core and liquid cladding. In (B), the curves are sigmoidal fits to guide the eye. The trend in (B) is counterintuitive (similar trend is seen in Figure S4 B), in that the lowest $\Delta \mathrm{n}$ systems experience comparable linewidthnarrowing at lower input powers than systems of higher $\Delta \mathrm{n}$. We believe that this effect is due to dye-solvent interaction, and not because "weak" waveguides make better lasers than "strong" waveguides (output powers are qualitatively similar for all $\Delta \mathrm{n}$ ).
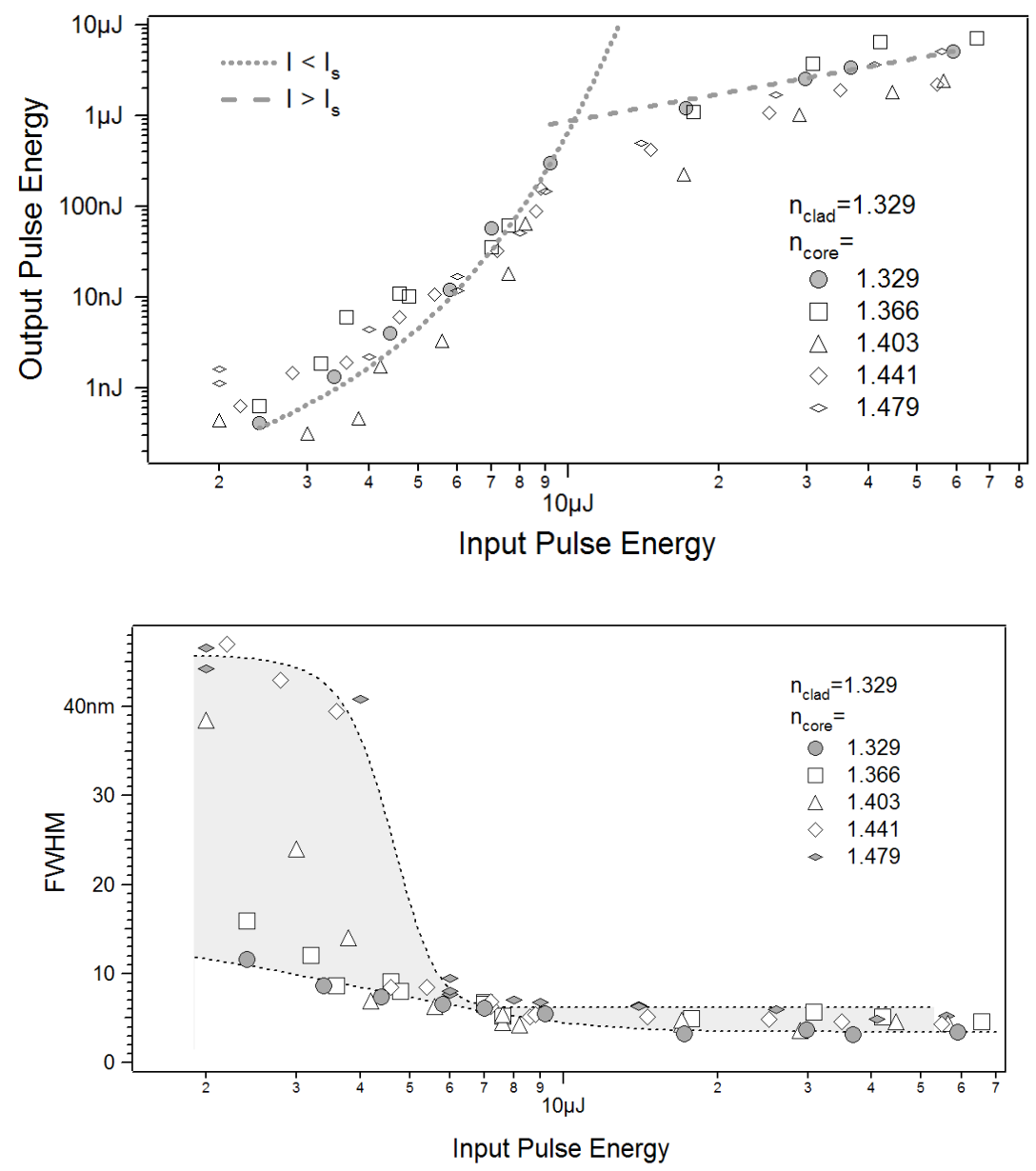
Figure S7. Output pulse energy versus input pulse energy for $\mathrm{L}^{2}$ waveguide laser containing 2-mM rhodamine 640 perchlorate (devices having terminal mirrors) having methanol as the core liquid and methanol as the cladding liquid. The front and back walls of the microfluidic channels are coated with Au layers (40 and $100 \mathrm{~nm}$ ).

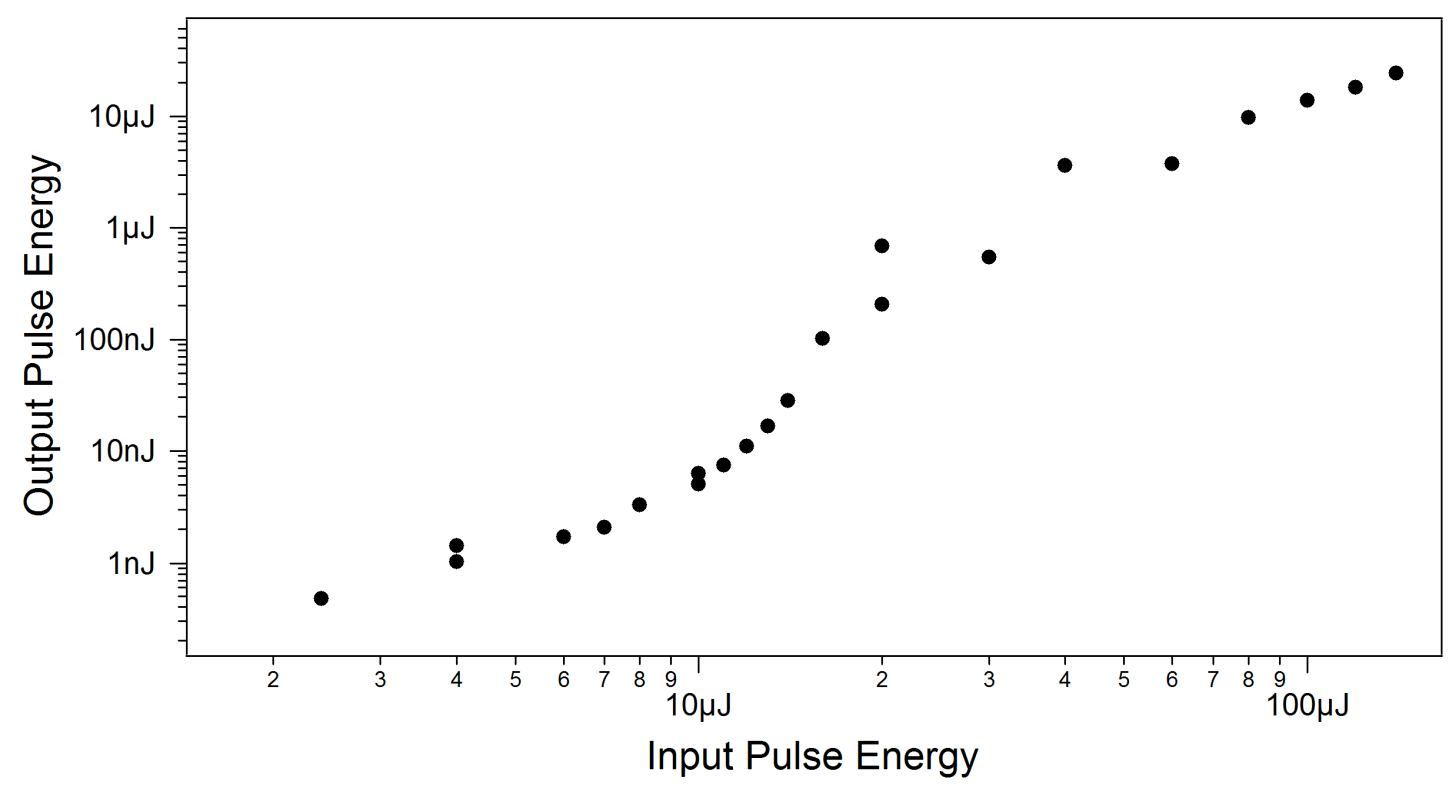

\section{References}

(1) Mayers, B. T.; Vezenov, D. V.; Vullev, V. I.; Whitesides, G. M. Anal. Chem. $\mathbf{2 0 0 5}$, in press.

(2) Vezenov, D. V.; Mayers, B. T.; Wolfe, D. B.; Whitesides, G. M. Appl. Phys. Lett. 2005, 86, 041104.

(3) Wolfe, D. B.; Conroy, R. S.; Garstecki, P.; Mayers, B. T.; Fischbach, M. A.; Paul, K. E.; Prentiss, M.; Whitesides, G. M. Proc. Nat. Acad. Sci. USA 2004, 101, 12434-12438.

(4) Svelto, O.; Taccheo, S.; Svelto, C. Optics Comm. 1998, 149, 277-282. 